\title{
BMJ Open Herbal medicines for the treatment of acute otitis media: protocol for a systematic review
}

\author{
Mi Ju Son, ${ }^{1}$ Yun Hee Kim, ${ }^{2}$ Young-Eun Kim, ${ }^{1}$ Hye Won Lee, ${ }^{2}$ Myeong Soo Lee ${ }^{3}$
}

To cite: Son MJ, Kim YH, Kim Y-E, et al. Herbal medicines for the treatment of acute otitis media: protocol for a systematic review. BMJ Open 2013;3 e003728. doi:10.1136/ bmjopen-2013-003728

- Prepublication history and additional material for this paper is available online. To view these files please visit the journal online (http://dx.doi.org/10.1136/ bmjopen-2013-003728).

MJS and YHK contributed equally.

Received 5 August 2013 Revised 20 October 2013 Accepted 22 October 2013

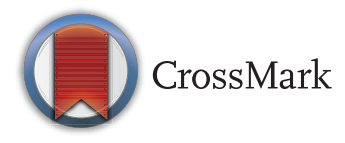

\footnotetext{
${ }^{1}$ Medical Culture \& Informatics Research Division, Korea Institute of Oriental Medicine, Daejeon, South Korea

${ }^{2}$ Herbal Medicine Research Division, Korea Institute of Oriental Medicine, Daejeon, South Korea

${ }^{3}$ Medical Research Division, Korea Institute of Oriental Medicine, Daejeon, South Korea
}

Correspondence to Myeong Soo Lee; drmslee@gmail.com

\section{ABSTRACT \\ Introduction: The aim of this systematic review is to analyse the trial data on the efficacy of herbal medicines for acute otitis media.}

Methods and analysis: The following 11 databases will be searched from their inception: MEDLINE, the Cumulative Index to Nursing and Allied Health Literature (CINAHL), EMBASE, Allied and Complementary Medicine Database (AMED), the Cochrane Central Register of Controlled Trials (CENTRAL), China Network Knowledge Infrastructure (CNKI) and five Korean databases (Oriental Medicine Advanced Searching Integrated System (OASIS), DBPIA, KoreaMed, Research Information Service System (RISS) and the Korean Studies Information Service System (KISS)). The selection of the studies, the data abstraction and the validations will be performed independently by two researchers.

Dissemination: The systematic review will be published in a peer-reviewed journal. The review will also be disseminated electronically and in print. Updates of the review will be conducted to inform and guide the healthcare practice and policy.

Trial registration number: PROSPERO 2013: CRD42013005100.

\section{INTRODUCTION}

\section{Description of the condition}

Acute otitis media (AOM) is one of the most common childhood infections, and has a high morbidity and low mortality. More than $80 \%$ of children experience AOM at least once, and one in three have more than three episodes. AOM has high socioeconomic costs because it is the most common cause of physicians' visits and requires a frequent diagnosis in prescribing the antibiotics for children. AOM indirectly causes the loss of children's time and caregivers' working time and income, in addition to the medical costs. According to the 2006 Health Medical Expenditure Panel Survey data, the yearly cost of AOM was about \$2.8-\$3.8 billion. ${ }^{1-4} \mathrm{AOM}$ is caused by bacterial infections, and the prevalence of nasopharyngeal colonisation by

\section{Strengths and limitations of this study}

- The strength of this systematic review is its extensive, unbiased search of various databases without a language restriction.

- The trial screening and data extraction will be conducted independently by two authors.

- Our systematic review may pertain to the potential incompleteness of the evidence reviewed, including publication and location bias, poor quality of the primary data and poor reporting of results.

organisms such as Streptococcus pneumoniae, Moraxella catarrhalis and non-typeable Haemophilus influenzae is strongly related to AOM. $^{5}$ AOM is defined as the presence of middle-ear effusion and the rapid onset of one or more signs or symptoms of middle-ear inflammation, such as an earache, ear rubbing, a bulging eardrum or fever. ${ }^{6}$ AOM can progress to recurrent $\mathrm{AOM}$, otitis media with effusion or conditions that are more serious, such as mastoiditis, meningitis, cerebritis and sigmoid sinus thrombosis. ${ }^{7}$

\section{Description of the intervention}

Although the majority of patients with $\mathrm{AOM}$ are treated with antibiotics, antibiotics have been suggested to provide only a marginal benefit and are not adequately effective for the relief of pain and distress. ${ }^{9}{ }^{10}$ A review of randomised controlled trials (RCT) involving the treatment of AOM with antibiotics revealed a limited role for antibiotics, and $80 \%$ of the untreated children were pain-free between 2 and 7 days after the onset of AOM. ${ }^{11} 12$

In recent years, a large number of AOM patients have chosen complementary and alternative medicine (CAM) to cure or prevent AOM. ${ }^{13}$ Forty-six per cent of children with recurrent $\mathrm{AOM}$ used some component of CAM such as herbal medicines. ${ }^{14}$ Herbal medicine is a therapy that utilises medicinal 
plants, minerals and animal parts to prevent or cure clinical conditions; it is approved by the WHO as a therapy for the treatment of AOM. ${ }^{15} 16$

\section{How the intervention might work}

Herbal medicines reduce the swelling and prevent endotoxin-induced otitis media by stimulating the function of the mucociliary system related to pathogen clearance in experimental research. ${ }^{17}{ }^{18}$ Also, many types of herbal medicines have anti-inflammatory and antibacterial effects, such as increasing the phagocytosis for clearing up an infection. ${ }^{8}{ }^{19-21}$ However, it is unclear how they work, in spite of observed immunomodulatory properties.

\section{Why it is important to do this review}

However, to our knowledge, no systematic reviews assessing the intervention of herbal drugs in AOM have been conducted to date despite this increasing use of CAM. A comprehensive evaluation of the efficacy and safety of herbal drugs will inform the recommendation for patients to use an herbal drug treatment.

\section{Objectives}

We will undertake a systematic review to assess the safety and efficacy of herbal drugs for the treatment of AOM.

\section{METHODS}

\section{Criteria for considering studies for this review}

Types of studies

Studies with the following study designs will be included:

1. RCTs, including cluster randomised trials;

2. Quasi-randomised trials, in which the group allocation is not purely random but may be determined by a factor such as a birth date, a hospital record number or alternation.

Any trials without parallel comparisons or control groups will be excluded.

\section{Types of participants}

Patients suffering from AOM, without ventilation tubes, will be included. The diagnostic criteria for AOM should be based on the criteria of the WHO, The American Academy of Pediatrics (AAP) and the American Academy of Otolaryngology and Head and Neck Surgery (AAOHNS), but if necessary, the trials with a definition of AOM used by the authors of the trial in question will be included.

\section{Types of interventions}

We will include those trials with the herbal medicine used alone or as a combined therapy of herbal medicine with a conventional therapy versus the same conventional therapy. Herbal medicine is defined as a single herb, an individually prescribed herbal formula or the herbal products extracted from the natural herbs. There is no limitation on the number of herbs used, the dosage, the forms of medication or the duration of the treatment. We will include only the oral administration of medication. Conventional therapy would include the usual medication such as antibiotic drugs, decongestants, antihistamines and topical analgesia. Surgery, however, would be excluded.

\section{Types of outcome measures}

\section{Primary outcome}

1. Proportion of patients with pain or intensity of pain

2. Proportion of patients with fever or intensity of fever

3. Proportion of patients with pain and fever or intensity of pain and fever

\section{Secondary outcome}

If possible, the following will be reviewed:

1. Abnormal tympanometry findings at various time points (4-6 weeks and 3 months) as a surrogate measure for hearing problems caused by middle-ear fluid

2. Tympanic membrane perforation

3. Contralateral otitis (in unilateral cases)

4. AOM recurrences

5. Serious complications related to AOM, such as mastoiditis and meningitis

6. Adverse effects likely to be related to herbal medicine

7. Quality of life

8. Duration of remission

\section{Search methods for the identification of studies}

\section{Electronic searches}

We will search for trials contained in the following electronic databases: MEDLINE, the Cumulative Index to Nursing and Allied Health Literature (CINAHL), EMBASE, Allied and Complementary Medicine Database (AMED) and the Cochrane Central Register of Controlled Trials (CENTRAL). We will also search one Chinese database (China Network Knowledge Infrastructure (CNKI)) and five Korean databases (Oriental Medicine Advanced Searching Integrated System (OASIS), DBPIA, KoreaMed, Research Information Service System (RISS) and the Korean Studies Information Service System (KISS)) .

\section{Searching other resources}

We will also check the reference lists of reviews and the retrieved articles for additional studies. We will search the metaRegister of Controlled Trials (mRCT; http://www. controlled-trials.com/mrct), Clinical trials.gov (http:// www.clinicaltrials.gov) and the WHO International Clinical Trials Registry platform (ICTRP) (http://apps. who.int/trialsearch/) for the ongoing trials.

\section{Search strategy}

Search strategies for The Cochrane Library, MEDLINE and EMBASE are presented in online supplementary appendix 1 . These strategies will be modified for use in other databases. 
Data collection and analysis

Selection of studies

Two review authors (MJS and YHK) will independently screen the titles and abstracts of the searched studies, perform the study selection and record their decisions on a standard eligibility form. When disagreements regarding the study selection are not resolved through discussions between these two authors, the arbiter (MSL) will decide.

\section{Inclusion criteria}

1. RCTs and Quasi-randomised trials

2. No language limitation

3. No publication status restriction

\section{Exclusion criteria}

1. Animal experiments

2. Non-RCTs

3. Case report/series, news items and letters

4. Qualitative studies

\section{Data extraction and management}

Two review authors (MJS and YHK) will read the hard copies of all the articles and independently extract the data using a standard data extraction form. Any disagreement between the authors will be resolved by a discussion with all the authors. When the reported data are insufficient or ambiguous, the authors (YHK and HWL) will contact the corresponding clinical trial authors through an email or telephone to request the additional information or clarification.

\section{Assessment of risk of bias in the included studies}

We will independently assess the risk of bias in the included studies according to the criteria from the Cochrane Handbook V.5.1.0, which include random sequence generation, allocation concealment, the blinding of participants and personnel, the blinding of outcome assessment, incomplete outcome data, selective reporting and other sources of bias. ${ }^{22}$ The quality of each trial will be categorised into a low, unclear or high risk of bias. If necessary, we will contact the authors of the assessed trials for clarification. We will resolve any differences in opinion through discussion or consultation with a third author.

\section{Measurement of the treatment effect}

For the continuous data, we will use the mean difference (MD) with 95\% CIs to measure the treatment effect. We will convert other forms of data into MDs. In the case of outcome variables with different scales, we will use the standard MD with $95 \%$ CIs. For dichotomous data, we will present the treatment effects as a relative risk (RR) with $95 \%$ CIs. We will convert other binary data into the RR form.
Units of analysis issues

For cross-over trials, data from the first treatment period will be used. For trials in which more than one control group was assessed, the primary analysis will combine the data from each control group. Subgroup analyses of the control groups will also be performed. Each patient will be counted only once in the analysis.

\section{Dealing with missing data}

Intention-to-treat analyses that include all the randomised patients will be performed. In the case of patients with missing outcome data, a carry-forward of the last observed response will be used. In the case where the individual patient data are unavailable they will be sought from the original source or from the published trial reports.

\section{Assessment of heterogeneity}

Clinically, various types of modalities and doses are included in herbal medicine treatments. We will use the random-effects model for the meta-analysis. If a meta-analysis is possible, we will use the $\mathrm{I}^{2}$ statistic for quantifying the inconsistencies among the included studies. According to the guidance given in the Cochrane Handbook for Systematic Reviews of Interventions, $50 \%$ will be the cut-off point for meaningful heterogeneity. If the heterogeneity is observed, we will conduct subgroup analysis to explore the possible causes. ${ }^{23}$

\section{Assessment of reporting biases}

If a sufficient number of included studies (at least 10 trials) are available, we will use the funnel plots to detect the reporting biases. ${ }^{24}$ However, the funnel plot asymmetry is not the same as the publication bias; therefore, we will attempt to distinguish the different possible reasons for the asymmetry, such as small-study effects, poor methodological quality and true heterogeneity of the included studies. ${ }^{24} 25$

\section{Data synthesis}

The differences between the intervention and the control groups will be assessed. RR and 95\% CIs will be assessed for the effect size of each included study. All statistical analyses will be conducted using the Cochrane Collaboration's software programme, Review Manager (RevMan), V.5.2.6 for Windows. For studies with insufficient information, we will contact the primary authors to acquire and verify the data when possible. The $\chi^{2}$ and the $\mathrm{I}^{2}$ tests will be used to evaluate the heterogeneity of the included studies. As soon as an excessive statistical heterogeneity is present, we will pool the data across the studies for a meta-analysis using a random effects model.

\section{Subgroup analysis and the investigation of heterogeneity}

If there are an adequate number of studies, we will conduct subgroup analyses to interpret the heterogeneity between the studies, including the following: 
1. Type of intervention

- Type of herbal medicines

- Existence of cotreatment, for example, herbal medicine used alone or as a combined therapy of herbal medicine with a conventional therapy

- The dose of herbal medicine

2. Type of design

- RCT or quasi RCT

3. Type of control

4. Type of age group

\section{Sensitivity analysis}

If a sufficient number of studies are available, sensitivity analyses will be conducted to determine whether the findings are robust.

1. Sample size (eg, more or less than 30 participants in each group)

2. Methodological qualities (eg, allocation concealment or the blinding of participants/assessors)

3. Analysis-related issues (eg, processes to handle the missing data)

\section{DISCUSSION}

As no primary data collection will be undertaken, no additional formal ethical assessment or informed consent is required. The systematic review will be published in a peerreviewed journal. It will also be disseminated electronically and in print. Updates of the review will be conducted to inform and guide the healthcare practice and policy.

Contributors The protocol was drafted by all authors. It was revised and the final version was approved by all authors.

Competing interests MJS was supported by the Korea Institute of Oriental Medicine (K13380), YHK and HWL were also supported by the same institute (K13101) and MSL was also supported by the same institute (K13400 and K13281).

\section{Provenance and peer review Not commissioned; internally peer reviewed.}

Open Access This is an Open Access article distributed in accordance with the Creative Commons Attribution Non Commercial (CC BY-NC 3.0) license, which permits others to distribute, remix, adapt, build upon this work noncommercially, and license their derivative works on different terms, provided the original work is properly cited and the use is non-commercial. See: http:// creativecommons.org/licenses/by-nc/3.0/

\section{REFERENCES}

1. Chu $\mathrm{CH}$, Wang MC, Lin LY, et al. Physicians are not adherent to clinical practice guidelines for acute otitis media. Int J Pediatr Otorhinolaryngol 2011;75:955-9.

2. Coker TR, Chan LS, Newberry SJ, et al. Diagnosis, microbial epidemiology, and antibiotic treatment of acute otitis media in children: a systematic review. JAMA 2010;304:2161-9.

3. Lee HJ, Park SK, Choi KY, et al. Korean clinical practice guidelines: otitis media in children. J Korean Med Sci 2012;27:835-48.

4. Marchisio P, Cantarutti L, Sturkenboom M, et al. Burden of acute otitis media in primary care pediatrics in Italy: a secondary data analysis from the Pedianet database. BMC Pediatr 2012;12:185.

5. Otsuka T, Kitami O, Kondo K, et al. Incidence Survey of Acute Otitis Media in Children in Sado Island, Japan-Sado Otitis Media Study (SADOMS). PLOS ONE 2013;8:e68711.

6. Damoiseaux RA, Rovers MM. AOM in children. Clin Evid (Online) 2011;2011:pii: 0301.

7. Grevers G, First International Roundtable ENTMG. Challenges in reducing the burden of otitis media disease: an ENT perspective on improving management and prospects for prevention. Int $J$ Pediatr Otorhinolaryngol 2010;74:572-7.

8. Maruyama Y, Hoshida S, Furukawa M, et al. Effects of Japanese herbal medicine, Juzen-taiho-to, in otitis-prone children-a preliminary study. Acta Otolaryngol 2009;129:14-18.

9. Ghalehbaghi B, Mohammadi N, Asghari A, et al. Evaluation of Iranian pediatric specialists' attitude and knowledge regarding approach to patients with acute otitis media. Med J Islam Repub Iran 2012;26:58-65.

10. Rovers MM, Glasziou P, Appelman CL, et al. Antibiotics for acute otitis media: a meta-analysis with individual patient data. Lancet 2006;368:1429-35.

11. Glasziou PP, Del Mar CB, Sanders SL, et al. Antibiotics for acute otitis media in children. Cochrane Database Syst Rev 2004;(1): CD000219.

12. Venekamp RP, Sanders S, Glasziou PP, et al. Antibiotics for acute otitis media in children. Cochrane Database Syst Rev 2013;(1): CD000219.

13. Lieberthal AS, Carroll AE, Chonmaitree $\mathrm{T}$, et al. The diagnosis and management of acute otitis media. Pediatrics 2013; 131:e964-99.

14. Marchisio P, Bianchini S, Galeone C, et al. Use of complementary and alternative medicine in children with recurrent acute otitis media in Italy. Int J Immunopathol Pharmacol 2011;24:441-9.

15. Barnes PM, Bloom B, Nahin RL. Complementary and alternative medicine use among adults and children: United States, 2007. Natl Health Stat Rep 2008;10:1-23.

16. Levi JR, Brody RM, McKee-Cole K, et al. Complementary and alternative medicine for pediatric otitis media. Int $J$ Pediatr Otorhinolaryngol 2013;77:926-31.

17. Sugiura $Y$, Ohashi $Y$, Nakai $Y$. The herbal medicine, sairei-to, prevents endotoxin-induced otitis media with effusion in the guinea pig. Acta Otolaryngol Suppl 1997;531:21-33.

18. Sugiura $Y$, Ohashi $Y$, Nakai $Y$. The herbal medicine, sairei-to, enhances the mucociliary activity of the tubotympanum in the healthy guinea pig. Acta Otolaryngol Suppl 1997;531:17-20.

19. Borchers AT, Sakai S, Henderson GL, et al. Shosaiko-to and other Kampo (Japanese herbal) medicines: a review of their immunomodulatory activities. J Ethnopharmacol 2000; 73:1-13.

20. Kim SH, Shin DS, Oh MN, et al. Inhibition of the bacterial surface protein anchoring transpeptidase sortase by isoquinoline alkaloids. Biosci Biotechnol Biochem 2004;68:421-4.

21. Xue CC, Hugel HM, Li CG, et al. Efficacy, chemistry and pharmacology of Chinese herbal medicine for allergic rhinitis. Curr Med Chem 2004;11:1403-21.

22. Higgins JPT, Altman DG, Sterne JAC. Chapter 8: assessing risk of bias in included studies. In: Higgins JPT, Green S. eds. Cochrane handbook for systematic reviews of interventions version 510 (updated March 2011) the Cochrane Collaboration. 2011. http://www. cochrane-handbook.org

23. Deeks JJ, Higgins JPT, Altman DG. Chapter 9: analysing data and undertaking meta-analyses. In: Higgins JPT, Green S. eds. Cochrane handbook for systematic reviews of interventions version 510 (updated March 2011) the Cochrane Collaboration. 2011. http://www.cochrane-handbook.org

24. Sterne JAC, Egger M, Moher D. Chapter 10: addressing reporting biases. In: Higgins JPT, Green S. eds. Cochrane handbook for systematic reviews of interventions version 510 (updated March 2011) the Cochrane Collaboration. 2011. http://www.cochrane-handbook.org

25. Egger M, Davey Smith G, Schneider M, et al. Bias in meta-analysis detected by a simple, graphical test. BMJ 1997; 315:629-34. 